\section{Stillen, wie es das Kind will}

Viele Mütter haben Bedenken, ihr Kind nicht ausreichend zu stillen. Häufig besteht Unsicherheit, wenn bestimmte Stillschemata nicht eingehalten werden können. Dr. Jacqueliene Kent, University of Western Australia, Perth, führte eine Beobachtungsstudie bei 71 Müttern durch, die ihr ein bis sechs Monate altes Kind ausschließlich stillten. Über 24 Stunden wurden dabei die Babys unmittelbar vor und nach dem Stillen gewogen und so die verabreichte Milchmenge bestimmt [Kent JC et al. Pediatrics 2006; 117: e387-95].

Zwischen dem Stillen mit einer Brust oder beiden Brüsten gab es keinen nennenswerten Unterschied (durchschnittlich verabreichte Milchmenge: 787 vs. $750 \mathrm{ml}$ pro 24 Stunden). Dabei war in der Regel eine Brust produktiver als die andere. 66\% der Säuglinge wurden mit der höchsten Milchmenge zwischen zehn Uhr abends und vier Uhr morgens ge-

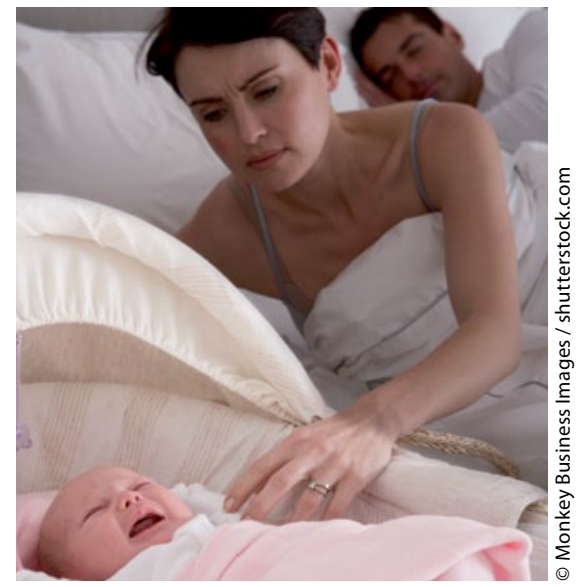

stillt („night feeders“), 34\% der Babys erhielten die meiste Milchmenge zwischen vier und zehn Uhr morgens ohne Stillen in der Nacht („day feeders“).

Die Milchproduktion lag im Durchschnitt bei $798 \mathrm{ml} / 24$ Stunden, hier war allerdings eine große Spannbreite zwi- schen 478 und $1.298 \mathrm{ml} / 24$ Stunden $\mathrm{zu}$ verzeichnen. Die Stillfrequenz lag bei elf pro Tag, wobei hier nicht die Anzahl der Mahlzeiten insgesamt, sondern der Stillvorgänge pro Brust gezählt wurde. Die mittlere Stillzeit betrug 15 Minuten, auch hier ergab sich eine große Variation zwischen fünf und 37 Minuten. Im Schnitt wurden pro Stillvorgang $75 \mathrm{ml}$ verabreicht. Der Fettanteil einer vollen Brust war niedriger als der einer (halb) entleerten Brust. Kent und ihre Mitarbeiter konnten aber zeigen, dass die Fettaufnahme des Säuglings unabhängig von der Stillfrequenz ist.

Kent unterstrich, dass es beim Stillen nicht auf das Erreichen von möglichst „normalen“ Volumina oder Laktationsfrequenzen ankommt; die Säuglinge bestimmen ihr Stillschema selbst. Die Messung über 24 Stunden konnte bei vielen Müttern das Vertrauen in das Stillen ihres Kindes stärken.

Kent J. Breastfeeding patterns

\section{Medikation der Mutter - was ist zu beachten?}

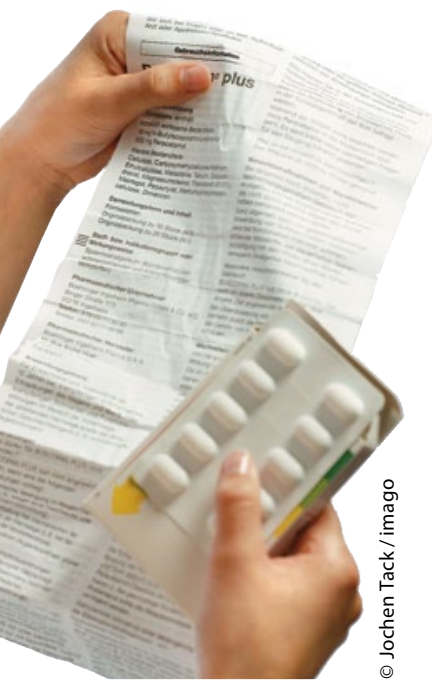

Bei den meisten Arzneimitteln besteht keine große Gefahr für das Kind durch das Stillen. Viele Medikamente mit großem Molekulargewicht, wie etwa Heparin, passieren nicht die Blut-MilchSchranke. Prof. Dr. Thomas Hale von der Texas Tech University in Lubbock, USA, ging auf die wichtigsten Substanzklassen ein: Zur Schwangerschaftsverhütung während der Stillzeit sollten Mütter östrogenhaltige Präparate nach Möglichkeit meiden, zu empfehlen ist die Minipille mit Progesteron. Bei den Antibiotika gelten Penicilline und Cephalosporine als sicher. Für Erythromycin und Azithromycin trifft dies mit Ausnahme der unmittelbar postpartalen Phase ähnlich $\mathrm{zu}$ - nach Behandlung von Säuglingen mit Erythromycin wurde allerdings ein erhöhtes Risiko für hypertrophe Pylorusstenosen beschrieben. Das Fluoroquinolon Ciprofloxacin sollte gemieden und dafür andere Substanzen wie Ofloxazin, Norfloxacin oder Levofloxacin eingesetzt werden. Bei allen Antibiotika ist jedoch deren ungünstiger Effekt auf die Darmflora zu beachten.

In der Gruppe der Antihypertensiva sind die Betablocker Atenolol und Acebutolol zu meiden, Substanzen wie Metoprolol und Propranolol sind hier günstiger. ACE-Hemmer sollten bei Frühgeborenen nicht eingenommen werden. Methyldopa und Hydralazin gelten als sehr sichere Medikamente, Kalziumkanalblocker (z.B. Nifedipin,
Nimodipin, Verapamil) werden ebenfalls bevorzugt. Als Schmerzmittel eignen sich nicht-steroidale Antirheumatika wie Ibuprofen oder Ketorolac. Bei den Opiaten sollte auf Pethidin verzichtet werden, Hydrocodon und Morphin sind insgesamt sichere Substanzen.

Hale warnte explizit vor radioaktivem Jod, das sich in hohen Konzentrationen in der Muttermilch anreichert. Alkaloide (beispielsweise in einigen Migränepräparaten) und Pseudoephedrin sollten nicht von stillenden Müttern eingenommen werden.

Hale betonte, dass eine Depression der Mutter sowohl für die Patientin als auch für ihr Kind gefährlich ist. Die meisten Antidepressiva sind sicher. Zwar sind Fälle etwa von Somnolenz unter Citalopram (bessere Alternative: Escitalopram) während der Laktation beschrieben, das Wichtigste jedoch ist eine erfolgreiche antidepressive Therapie: „Use the one that works!"

Hale T. Medication and mother's milk

Berichterstattung: Dr. Andreas Fischer, München 